\title{
Sphingomyelins in Four Ascidians, Ciona intestinalis, Halocynthia roretzi, Halocynthia aurantium, and Styela clava
}

\author{
Masahiro Ito ${ }^{*}$, Kazuhito Yokoi ${ }^{1}$, Takashi Inoue ${ }^{1}$, Shogo Asano ${ }^{1,3}$, Rei Hatano ${ }^{1}$, \\ Ryota Shinohara ${ }^{1,4}$, Saki Itonori ${ }^{2}$ and Mutsumi Sugita ${ }^{2}$ \\ ${ }^{1}$ Department of Bioinformatics, Institute of Science and Engineering, Ritsumeikan University (1-1-1 Nojihigashi, Kusatsu, Shiga 525-8577, \\ JAPAN) \\ ${ }^{2}$ Department of Chemistry, Faculty of Liberal Arts and Education, Shiga University (2-5-1 Hiratsu, Ohtsu, Shiga 520-0802, JAPAN) \\ ${ }^{3}$ Graduate School of Frontier Biosciences, Graduate School of Medicine, Osaka University (2-2 Yamadaoka, Suita, Osaka 565-0871, \\ JAPAN) \\ ${ }^{4}$ Department of Pharmacology, Kyoto University Faculty of Medicine (Konoe-cho, Yoshida, Sakyo-ku, Kyoto 606-8501, JAPAN)
}

\begin{abstract}
Sphingomyelin is rarely found in lower animals, while sphingophospholipid is a characteristic of higher animals. In this study, sphingomyelin was first isolated and characterized from ascidian Ciona intestinalis. Ascidian sphingomyelin was prepared using ion exchange (QAE-Sphadex-A25) and silicic acid (Florisil and Iatrobeads) column chromatographies. The chemical structure was characterized by fatty acid analysis, sphingoid analysis, hydrogen fluoride degradation, acid hydrolysis, enzymatic hydrolysis, infrared analysis, and matrix-assisted laser desorption/ionization time-of-flight mass spectrometry. The ceramide moieties of $C$. intestinalis sphingomyelin consisted primarily of C16:0, C18:0, and C18:1 fatty acids and d18:2 sphingadiene. Furthermore, sphingomyelins were isolated and characterized from 3 other ascidians, Halocynthia roretzi, Halocynthia aurantium, and Styela clava using the same methods. Comparative analysis of the sphingomyelin structures in $\mathbf{4}$ ascidian species- $C$. intestinalis (Enterogona) and $H$. roretzi, H. aurantium, and S. clava (Pleurogona)-revealed that the major fatty acid composition of the ceramides was similar, and that they differed in minor components.
\end{abstract}

Key words: sphingomyelin, phosphosphingolipid, ascidian, comparative analysis, ceramide

\section{INTRODUCTION}

Sphingomyelin, a major sphingolipid in cellular membranes, is a ubiquitous lipid in vertebrates. Sphingomyelin is characterized by a ceramide unit, which consists of a sphingoid and a fatty acid, along with a phosphorylcholine. On the structural basis, it is the analog of phosphatidylcholine. Sphingomyelin serves primarily as a structural component in cellular membranes, recently, sphingomyelin-derived second messengers important regulatory molecules of intracellular signal transductions have been reported; for example, ceramides have been shown to suppress mitogenesis, while sphingosines and sphingosine 1phosphates have been known to enhance cell growth ${ }^{1,2)}$. However, the intracellular signal pathways are currently unclear.
On the other hand, it was believed that sphingomyelin is not a ubiquitous sphingolipid in invertebrates. While analyzing ceramide phosphoethanolamine composition in a pound-snail, Heterogen longispira ${ }^{3,4}$ and a green-bottle fly, Lucilia caesar ${ }^{5}$, sphingomyelin was not detected. In 1996, sphingomyelin was characterized from a parasitic nematode, Ascaris sum, in invertebrate ${ }^{6)}$. Recently, sphingomyelin was identified in invertebrates by conducting a mobility analysis and was detected by a chemical reagent using thin-layer chromatography (TLC). In addition, sphingomyelin was detected by analyzing the ceramide cholinephosphotransferase gene and by using a sphingomyelinspecific toxin-lysenin-extracted from the coelomic fluid of earthworms ${ }^{7,8}$. However, these methods have been unsuccessful for isolating sphingomyelin and analyzing its

*Correspondence to: Masahiro Ito, Department of Bioinformatics, Institute of Science and Engineering, Ritsumeikan University, 1-1-1 Nojihigashi, Kusatsu, Shiga 525-8577, JAPAN

E-mail: maito@sk.ritsumei.ac.jp

Accepted April 28, 2009 (recieved for review February 6, 2009)

Journal of Oleo Science ISSN 1345-8957 print / ISSN 1347-3352 online

http://www.jstage.jst.go.jp/browse/jos/ 
ceramide composition.

Ascidian is a model organism for evaluating the characteristics of vertebrates because like vertebrates, it has a notochord, which is a precursor of the backbone of a chordate. Moreover, the complete genome sequence of ascidian $C$. intestinalis has already been determined ${ }^{9}$; a large amount of genome-related data has been accumulated using such as EST analysis and in situ hybridization ${ }^{10,11}$. Research on ascidians has advanced in the post-genome era, with a focus on glycans and lipids. Recently, 2 novel acidic glycosphingolipids were detected in 2 ascidians- $C$. intestinalis and $H$. roretzi $i^{12,13)}$. Although ascidian phospholipid has not yet been isolated, sphingomyelin has been estimated on the basis of binding of lysenin to sphingomyelin.

We describe the structural characterization of sphingomyelin from an ascidian of Enterogona, C. intestinalis, and 3 other ascidian species of Pleurogona, Halocynthia roretzi, Halocynthia aurantium, and Styela clava, and the comparison of the ceramide composition of these sphingomyelins. To understand sphingomyelin and sphingomyelin-derived second messenger functions, it is first necessary to compare them in isolation and characterize sphingomyelins.

\section{EXPERIMENTAL}

\subsection{Materials}

C. intestinalis was collected at Kobe Port near Kobe University in Japan. H. roretzi, H. aurantium, and S. clava were purchased from Hiroyo Fisheries Ltd., Nishikiya Saito Ltd., and SEA Kukaku Corp., respectively, in Japan.

\subsection{Extraction of sphingomyelins}

After the cellulose crusts were removed from three specimens, except $C$. intestinalis, these specimens were boiled, suspended in acetone, and dried naturally. The dry specimens were homogenized using a blender and extracted twice with chloroform-methanol $(2: 1, \mathrm{v} / \mathrm{v})$ for $2 \mathrm{~h}$ and once with chloroform-methanol $(1: 1, \mathrm{v} / \mathrm{v})$ overnight at room temperature. The extracts were combined and concentrated using a rotary evaporator at $40^{\circ} \mathrm{C}$. The crude lipid fraction was subjected to mild alkaline hydrolysis in $0.5 \mathrm{M} \mathrm{KOH}$ in methanol/water $(95: 5, \mathrm{v} / \mathrm{v})$ at $37^{\circ} \mathrm{C}$ overnight to eliminate acyl and alkenyl glycerolipids for obtaining sphingolipids. The alkali-stable product that resulted in a precipitate by cold acetone was applied to a QAE-Sephadex A-25 (GE Healthcare Bio-Sciences) column chromatography (column size, $3.4 \mathrm{~cm} \times 22 \mathrm{~cm}$ ) and eluted with 3 volumes of chloroform-methanol-water (30:60:8, v/v/v) for neutral and zwitterionic sphingolipids fraction including sphingomyelins, and with 5 volumes of $0.05 \mathrm{M}, 3$ volumes of $0.15 \mathrm{M}$, and 3 volumes of $0.45 \mathrm{M}$ ammonium acetate in methanol for the extraction of acidic sphingolipids fraction, respectively. The obtained neutral sphingolipids fraction including sphingomyelins was concentrated and dried by a rotary evaporator and a $\mathrm{N}_{2}$ evaporator.

\subsection{Isolation and purification of sphingomyelin}

The obtained neutral sphingolipids fraction was acetylated by adding pyridine/acetic anhydride (2:3, v/v) and reacted at $20^{\circ} \mathrm{C}$ for $18 \mathrm{~h}$. The acetylated neutral sphingolipids fraction was applied to a Florisil (magnesium silicate; Nacalai Tesque) column chromatography (column size 1.8 $\mathrm{cm} \times 100 \mathrm{~cm}$ ) to remove neutral glycosphingolipids. Three volumes of each 1,2-dichloroethane-methanol (3:1, v/v), 1,2dichloroethane-methanol-water (20:80:10, v/v/v), and chloroform-methanol-water $(60: 40: 10, \mathrm{v} / \mathrm{v} / \mathrm{v})$ were eluted to obtain the acetylated neutral phosphosphingolipid fraction including sphingomyelins. This acetylated neutral phosphosphingolipid fraction (1,2-dichloroethane-methanolwater fraction) was deacetylated by $0.5 \mathrm{M}$ potassium hydroxide in methanol at $37^{\circ} \mathrm{C}$ for $12 \mathrm{~h}$, and neutralized by the addition of an appropriate amount of hydrochloric acid and then dialyzed. This fraction was chromatographed to remove a phospholipid except a phospoglycerolipid of glycerol-ether type, on an Iatrobeads (silica gel 6RS-8060; Mitsubishi Kagaku Iatron, Inc.) column (column size, $2.0 \mathrm{~cm} \times$ $20 \mathrm{~cm}$ ) with chloroform-methanol-water $(60: 25: 8, \mathrm{v} / \mathrm{v} / \mathrm{v})$. The effluents were fractionated every $5 \mathrm{ml}$ into a test tube using a fraction-collector and monitored by TLC. The collected sphingomyelin fraction was also confirmed by infrared absorption spectroscopy.

\subsection{Thin-layer chromatography (TLC)}

The samples were monitored by TLC using silica gel 60 plates (Merck, Germany). As running solvents, sphingomyelin, lyso-sphingomyelin (sphingosylphosphorylcholine), and ceramide were developed with chloroformmethanol-water (60:40:10, v/v/v), chloroform-methanolwater-acetic acid (60:40:10:10, v/v/v/v), and chloroformmethanol (92:8, v/v), respectively. The sphingomyelins were visualized by spraying with the Dittmer-Lester reagent ${ }^{14)}$ and the Hanes-Isherwood reagent ${ }^{15)}$ for phosphorus, ninhydrin reagent for free amino groups and 50\%$\mathrm{H}_{2} \mathrm{SO}_{4}$ regent for ceramides. Cellulose thin-layer plates were used for the TLC of the phosphocholine (Funakoshi Corp.), which was developed using butanol-acetic acidwater (40:10:20, v/v/v) as a running solvent, and visualized using the Hanes-Isherwood regent for phosphorus.

\subsection{Infrared absorption spectroscopy}

The sample $(\sim 100 \mu \mathrm{g})$ dissolved as $1 \mathrm{mg} / \mathrm{mL}$ concentration with chloroform-methanol $(2: 1, \mathrm{v} / \mathrm{v})$ was dried on the crystal plate and analyzed using Fourier transform infrared absorption spectrophotometer, Shimadzu FTIR8400S equipped with MIRacle ${ }^{\mathrm{TM}} \mathrm{A}$. 


\subsection{Butanol-hydrochloric acid hydrolysis}

To obtain hydrolyzates including lyso-sphingomyelin and fatty acids from sphingomyelin, acid hydrolysis was carried out by treatment of the sphingomyelins $(2 \mathrm{mg})$ with 6 $\mathrm{M}$ butanol-hydrochloric acid $(0.5 \mathrm{~mL})$ at $100^{\circ} \mathrm{C}$ for $1 \mathrm{~h}$. These hydrolyzates were washed in acetone three times to remove free fatty acids. The remaining hydrolyzates, crude lyso-sphingomyelin, were chromatographed on an Iatrobeads column (column size, $1.0 \mathrm{~cm} \times 12 \mathrm{~cm}$ ) by stepwise elution with chloroform-methanol (80:20, v/v, 70:30, $\mathrm{v} / \mathrm{v}$, and $60: 40, \mathrm{v} / \mathrm{v}$ ) and chloroform-methanol-water $(60: 40: 10, \mathrm{v} / \mathrm{v} / \mathrm{v})$. The obtained purified lyso-sphingomyelin was analyzed by infrared absorption spectroscopy and TLC with chloroform-methanol-acetic acid-water (60:40:10:10, $\mathrm{v} / \mathrm{v} / \mathrm{v})$ as a running solution.

\subsection{Hydrogen fluoride degradation}

The sample $(2 \mathrm{mg})$ was dissolved in $0.5 \mathrm{~mL}$ of dimethylsulfoxide and hydrogen fluoride was added to this lysate, and then allowed to stand at room temperature for $20 \mathrm{~h}$. To obtain the ceramide fraction, this solution was dialyzed with running water and freeze-dried. The obtained ceramide fraction was analyzed by TLC with chloroformmethanol $(92: 8, \mathrm{v} / \mathrm{v})$ as a running solution.

\subsection{Enzymatic hydrolysis}

Sphingomyelin $(2 \mathrm{mg})$ was dissolved in $0.2 \mathrm{ml}$ of $50 \mathrm{mM}$ Tris- $\mathrm{HCl}\left(\mathrm{pH}\right.$ 7.5) buffer with $0.1 \mathrm{~mL}$ of $20 \mathrm{mM} \mathrm{CaCl}_{2}$ and $0.2 \mathrm{~mL}$ of ethyl-ether, and was then enzymatically hydrolyzed by phospholipase $\mathrm{C}$ in $0.1 \mathrm{~mL}$ of $50 \mathrm{mM}$ Tris$\mathrm{HCl}(\mathrm{pH} 7.5)$ buffer at $37^{\circ} \mathrm{C}$ for $34 \mathrm{~h}$. The hydrolyzed sphingomyelin solution was heated to remove ethyl-ether and 2 $\mathrm{mL}$ of chloroform-water $(2: 1, \mathrm{v} / \mathrm{v})$ was added to separate using the Folch partition. The lower solution was dried and analyzed by TLC with chloroform-methanol (92:8, v/v) as a running solution.

\subsection{Ceramide composition analysis}

Sphingoids were prepared from sphingomyelins by methanolysis with $1 \mathrm{M}$ aqueous methanolic $\mathrm{HCl}$ at $70^{\circ} \mathrm{C}$ for $18 \mathrm{~h}$. The $O$-trimethylsilyl derivatives of sphingoids were analyzed using the Shimadzu GC-18A gas chromatograph with the Shimadzu HiCap-CBP5 $(0.22 \mathrm{~mm} \times 25 \mathrm{~m})$ programmed at $2^{\circ} \mathrm{C} / \mathrm{min}$ from $210^{\circ} \mathrm{C}$ to $230^{\circ} \mathrm{C}$. Fatty acids were prepared from sphingomyelins by hydrolysis with $1 \mathrm{M}$ aqueous methanolic $\mathrm{HCl}$ at $100^{\circ} \mathrm{C}$ for $3 \mathrm{~h}$. The fatty acid methyl esters were extracted three times using $0.5 \mathrm{~mL}$ of n-hexane and then analyzed using the Shimadzu GC-18A gas chromatograph with the Shimadzu HiCap-CBP5 (0.22 $\mathrm{mm} \times 25 \mathrm{~m}$ ) programmed at $2^{\circ} \mathrm{C} / \mathrm{min}$ from $170^{\circ} \mathrm{C}$ to $230^{\circ} \mathrm{C}$.

\subsection{MALDI-TOF MS analysis}

The sphingomyelin was prepared as a $1 \mathrm{mg} / \mathrm{mL}$ solution in chloroform/methanol $(2: 1, \mathrm{v} / \mathrm{v})$. It was separately put on a plate in the order of sphingomyelin and matrix solution $(1: 1, \mathrm{v} / \mathrm{v} ; 4 \mu \mathrm{g} / \mu \mathrm{L})$, and was crystallized by natural drying. The matrix used $\alpha$-CHCA ( $\alpha$-Cyano-4-hydroxycinnamic acid; Wako Pure Chem. Industries Ltd. Japan). The MS spectra were calibrated externally using a standard peptide calibration mixture containing $1 \mathrm{mg} / \mathrm{mL}$ each of angiotensin I (1,296.68 Mass Units; Wako Pure Chem. Industries Ltd. Japan) and bradykinin fragments 1-5 (573.31 Mass Units; Sigma Chemical Co.). MALDI-TOF MS analysis was performed using an Applied Biosystems/Voyager$\mathrm{DE}^{\mathrm{TM}}$ STR Biospectrometer with a nitrogen laser (337 nm) and an acceleration voltage of $20 \mathrm{kV}$, operating in the reflector positive ion mode.

\section{RESULTS}

\subsection{Isolation of sphingomyelin from $C$. intestinalis}

By using chloroform/methanol solution, lipid (14.0 g) was extracted from the dried powder $(400.0 \mathrm{~g})$ of the ascidian $C$. intestinalis, was treated to mild-alkali hydrolysis. The obtained alkali-stable substances (7.85 g) were applied to a QAE-Sephadex column. The lipid fraction $(3.0 \mathrm{~g})$ including sphingomyelin was recovered from the QAE-Sephadex column with chloroform/methanol/water as the pass-through fraction (Fig. 1(A)). Then, this lipid fraction was purified by Florisil column chromatography after acetylation. The phosphosphingolipid fraction including sphingomyelin was deacetylated and returned to the original state $(373 \mathrm{mg})$. Finally, $27.3 \mathrm{mg}$ of sphingomyelin was isolated from 50.0 mg of phosphosphingolipid fraction by Iatrobeads column chromatography with chloroform/methanol/water (60:25:3, v/v/v) (Fig. 1(B)).

\subsection{Identification of ceramide, phosphocoline, and sphin- gosyl-phosphocoline}

Sphingomyelin of $C$. intestinalis was identified by the following analyses.

3.2.1 Infrared absorption spectroscopy analysis

The sphingomyelin of $C$. intestinalis was analyzed using IR. As a result, three sphingomyelin-specific absorptions, that is, the amide bond $\left(1,550 \mathrm{~cm}^{-1}\right.$ and $\left.1,650 \mathrm{~cm}^{-1}\right)$, phosphorus-oxygen bond $\left(1,220 \mathrm{~cm}^{-1}\right)$, and choline group (960 $\mathrm{cm}^{-1}$ ) were observed (Fig. 2(1)).

3.2.2 Butanol-hydrochloric acid hydrolysis

Lyso-sphingomyelin is produced from sphingomyelin by the butanol-hydrochloric acid hydrolysis. The resolvent produced from sphingomyelin from $C$. intestinalis by butanol-hydrochloric acid hydrolysis was analyzed by TLC and IR spectroscopy. Sphingomyelin shows a positive reaction only to the Dittmer-Lester reagent but the resolvent after hydrolysis showed positive reactions to both DittmerLester and ninhydrin reagents (data not shown). In the IR analysis, no amide bond absorption of the resolvent was 

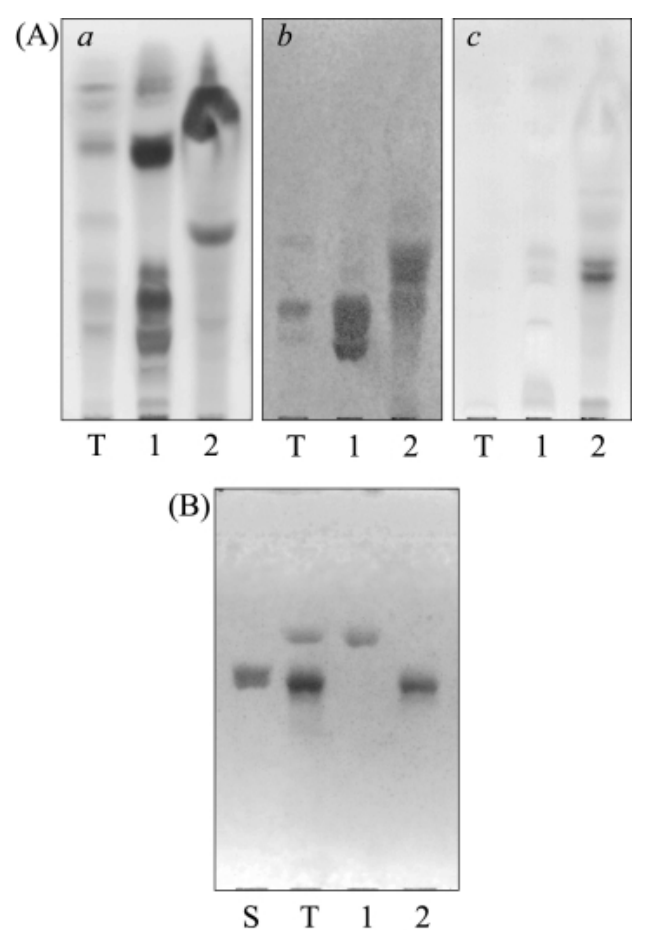

Fig. 1 TLC Analysis after QAE Sephadex-A25 (A) and Iatrobeads (B) Column Chromatographies.

(A): TLC was performed using chloroformmethanol-water $(60: 40: 10, \mathrm{v} / \mathrm{v} / \mathrm{v})$ and visualized by $a$ : orcinol $/ \mathrm{H}_{2} \mathrm{SO}_{4}, b$ : Hanes-Isherwood, and $c$ : ninhydrin staining; lane $\mathrm{T}$, faction before QAE Sephadex-A25 column chromatography; lane 1, non absorption; lane 2, absorption, (B): TLC was performed using chloroform-methanol-water $(60: 40: 10, \mathrm{v} / \mathrm{v} / \mathrm{v})$ and visualized by HanesIsherwood staining, lane $\mathrm{S}$, sphingomyelin from bovine brain; lane $\mathrm{T}$, fraction before Iatrobeads column chromatography; lane 1, isolated sphingomyelin; lane 2, phospholipid except the sphingomyelin.

observed in comparison with sphingomyelin (Fig. 2 (2)). 3.2.3 Hydrogen fluoride degradation analysis

Ceramide is produced from sphingomyelin by hydrogen fluoride degradation treatment. The obtained reaction products by hydrogen fluoride treatment were purified by Iatorobeads chromatography. The purified product was analyzed by TLC and IR spectroscopy. By TLC analysis, the band of the product migrated to a similar position as with that of a standard ceramide (data not shown). In addition, the IR spectra of the product did not show absorptions of choline group and the hydroxyl group of phosphate, but did show the absorption of amide bond from ceramide (Fig. 2(3)).

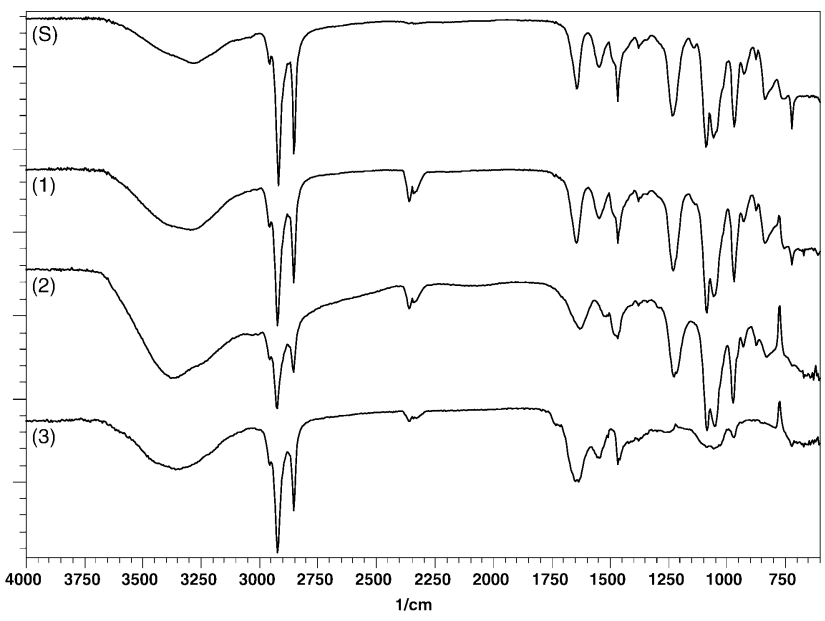

Fig. 2 Infrared Spectra of Sphingomyelin.

(S) standard sphingomyelin from bovine brain, (1) intact sphingomyelin from C. intestinalis, (2) butanol-hydrochloric acid hydrolyzed sphingomyelin, and (3) hydrogen fluoride degraded sphingomyelin.
(A)

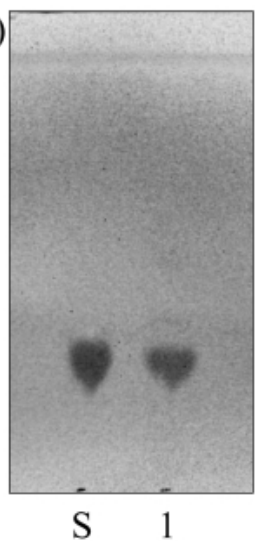

(B)



Fig. 3 TLC Analysis after Phospholipase C Treatment.

TLC was performed using chloroform-methanol (92:8, v/v), and visualized by Hanes-Isherwood staining (A); lane S, phosphocoline from bovine brain; lane 1, lower portion from $C$. intestinalis, and $50 \% \mathrm{H}_{2} \mathrm{SO}_{4}$ staining (B), lane $\mathrm{S} 1$, normal-type ceramide from bovine brain; lane S2, hydroxyl-type ceramide from bovine brain; lane 1, upper portion from C. intestinalis.

\subsubsection{Enzymatic hydrolysis}

A phosphocholine and a ceramide were produced by the phospholipase $\mathrm{C}$ treatment (Fig. 3 ). The reaction products by phospholipase C hydrolysis were purified by Iatorobeads chromatography. On TLC analysis, the puri- 
fied product showed the same $\mathrm{Rf}$ value as that of ceramide obtained by the hydrogen fluoride treatment.

\subsection{Ceramide and sphingoid analysis}

The ceramide moiety of $C$. intestinalis sphingomyelin was characterized by CG and CG-MS analyses (Table 1). The fatty acids were mainly composed of $\mathrm{C} 16: 0, \mathrm{C} 18: 0$, and C18:1 and the sphingoids were composed entirely of d16:1 sphingenine and d18:2 sphingadiene.

\subsection{MALDI-TOF MS analysis}

The putative structure of the sphingomyelin purified from $C$. intestinalis was confirmed by MALDI-TOF MS analysis in the reflector positive-ion mode (Fig. 4). A sum-

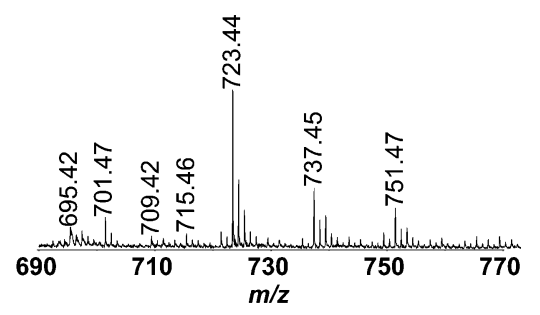

Fig. 4 MALDI-TOF MS Analysis of C. intestinalis Sphingomyelin.

Sphingomyelin obtained after Iatrobeads column separation were monitored as $[\mathrm{M}+\mathrm{H}]^{+}$and $[\mathrm{M}+\mathrm{Na}]^{+}$ by MALDI-TOF MS with $\alpha$-CHCA as a matrix in the positive-ion mode.

Table 1 Ceramide Moiety of Sphingomyelin.

(A) Fatty Acid

\begin{tabular}{|c|c|c|c|c|}
\hline & Enterogona & & Pleurogona & \\
\hline Fatty Acid & $\begin{array}{c}\text { Ciona } \\
\text { intestinalis }\end{array}$ & $\begin{array}{c}\text { Halocynthia } \\
\text { roretzi }\end{array}$ & $\begin{array}{c}\text { Halocynthia } \\
\text { aurantium }\end{array}$ & $\begin{array}{l}\text { Styela } \\
\text { clava }\end{array}$ \\
\hline $14: 0$ & 3.3 & 4.1 & 4.9 & - \\
\hline $15: 0$ & - & 6.1 & 4.6 & - \\
\hline $16: 0$ & 53.7 & 25.4 & 18.1 & 15.1 \\
\hline $17: 0$ & 6.5 & 7.2 & 6.5 & 12.1 \\
\hline $18: 0$ & 12.8 & 16.8 & 18.6 & 4.1 \\
\hline 19:0 & - & - & 2.3 & 5.3 \\
\hline $20: 0$ & 2.5 & 2.5 & 5.0 & 14.8 \\
\hline $21: 0$ & 2.2 & - & - & 8.1 \\
\hline $22: 0$ & 7.5 & - & 25.8 & 17.8 \\
\hline $23: 0$ & - & - & 4.5 & 5.0 \\
\hline $24: 0$ & - & - & 2.1 & 2.9 \\
\hline $16: 1$ & - & 2.1 & - & - \\
\hline $18: 1$ & 11.4 & - & - & - \\
\hline $22: 1$ & - & 4.1 & - & - \\
\hline h14:0 & - & 2.5 & - & - \\
\hline h15:0 & - & 2.1 & - & - \\
\hline h16:0 & - & 16.7 & 2.2 & 2.8 \\
\hline h17:0 & - & 3.1 & - & 4.3 \\
\hline h18:0 & - & 7.2 & 2.6 & - \\
\hline h19:0 & - & - & 3.0 & 3.1 \\
\hline h20:0 & - & - & - & 4.7 \\
\hline
\end{tabular}

(B) Sphingoid

\begin{tabular}{ccccc}
\hline & $\begin{array}{c}\text { Enterogona } \\
\text { Ciona }\end{array}$ & Halocynthia & Pleurogona & Halocynthia \\
roretzi & aurantium & $\begin{array}{c}\text { Styela } \\
\text { clava }\end{array}$ \\
\hline d16:1 & 8.8 & 37.9 & 32.9 & 29.3 \\
d18:2 & 91.2 & 62.1 & 67.1 & 70.7 \\
\hline
\end{tabular}


Table 2 Summary of MALDI-TOF MS Analysis in C. intestinalis.

\begin{tabular}{cccc}
\hline Measured & Calculated & Ionization & $\begin{array}{c}\text { Composition } \\
\text { (Sphingoid-Fatty Acid) }\end{array}$ \\
\hline 695.42 & 695.51 & {$[\mathrm{M}+\mathrm{Na}]^{+}$} & $\mathrm{d} 18: 2-\mathrm{C} 14: 0$ \\
701.47 & 701.56 & {$[\mathrm{M}+\mathrm{H}]^{+}$} & $\mathrm{d} 16: 1-\mathrm{C} 18: 1, \mathrm{~d} 18: 2-\mathrm{C} 16: 0$ \\
709.42 & 709.53 & {$[\mathrm{M}+\mathrm{Na}]^{+}$} & $\mathrm{d} 18: 2-\mathrm{C} 15: 0$ \\
715.46 & 715.58 & {$[\mathrm{M}+\mathrm{H}]^{+}$} & $\mathrm{d} 18: 2-\mathrm{C} 17: 0$ \\
723.44 & 723.54 & {$[\mathrm{M}+\mathrm{Na}]^{+}$} & $\mathrm{d} 16: 1-\mathrm{C} 18: 1, \mathrm{~d} 18: 2-\mathrm{C} 16: 0$ \\
737.45 & 737.56 & {$[\mathrm{M}+\mathrm{Na}]^{+}$} & $\mathrm{d} 18: 2-\mathrm{C} 17: 0$ \\
751.47 & 751.57 & {$[\mathrm{M}+\mathrm{Na}]^{+}$} & $\mathrm{d} 18: 2-\mathrm{C} 18: 0$ \\
\hline
\end{tabular}

Table 3 Summary of Yields of Each Fraction and Sphingomyelin.

\begin{tabular}{|c|c|c|c|c|}
\hline & Enterogona & & Pleurogona & \\
\hline & $\begin{array}{c}\text { Ciona } \\
\text { intestinalis }\end{array}$ & $\begin{array}{c}\text { Halocynthia } \\
\text { roretzi }\end{array}$ & $\begin{array}{c}\text { Halocynthia } \\
\text { aurantium }\end{array}$ & $\begin{array}{l}\text { Styela } \\
\text { clava }\end{array}$ \\
\hline Wet weight & $10.0 \mathrm{~kg}\left(3.33 \times 10^{3}\right)$ & $5.6 \mathrm{~kg}\left(3.29 \times 10^{3}\right)$ & $7.0 \mathrm{~kg}\left(1.56 \times 10^{3}\right)$ & $3.5 \mathrm{~kg}\left(5.00 \times 10^{3}\right)$ \\
\hline Dry weight & $0.4 \mathrm{~kg}\left(1.33 \times 10^{2}\right)$ & $0.3 \mathrm{~kg}\left(1.76 \times 10^{2}\right)$ & $1.7 \mathrm{~kg}\left(3.78 \times 10^{2}\right)$ & $0.3 \mathrm{~kg}\left(4.29 \times 10^{2}\right)$ \\
\hline $\mathrm{C} / \mathrm{M}$ extract & $40.0 \mathrm{~g}(13.3)$ & $41.9 \mathrm{~g} \mathrm{(24.7)}$ & $218.5 \mathrm{~g}(48.6)$ & $22.8 \mathrm{~g}(32.6)$ \\
\hline Sphingolipid & $7.9 \mathrm{~g}(2.63)$ & $3.3 \mathrm{~g}(1.94)$ & $10.4 \mathrm{~g}(2.31)$ & $3.8 \mathrm{~g}(5.43)$ \\
\hline Neutral lipid & $3.0 \mathrm{~g}(1.00)$ & $1.7 \mathrm{~g}(1.00)$ & $4.5 \mathrm{~g}(1.00)$ & $0.7 \mathrm{~g}(1.00)$ \\
\hline Phospholipid & $373.0 \mathrm{mg}(0.12)$ & $147.8 \mathrm{mg}(0.09)$ & $163.1 \mathrm{mg}(0.04)$ & $133.2 \mathrm{mg}(0.19)$ \\
\hline Sphingomyelin & $203.7 \mathrm{mg}(0.07)$ & $93.7 \mathrm{mg}(0.06)$ & $128.7 \mathrm{mg}(0.03)$ & - \\
\hline
\end{tabular}

Here, the values in the parenthesis show each ratio for neutral lipid 1.0.

mary of MALDI-TOF MS analysis is shown in Table 2. The major peaks were found at $\mathrm{m} / z 701.47,723.44,737.45$, and 751.47 and the minor peaks were found at $\mathrm{m} / z$ 695.42, 701.47, 709.42, and 715.46 (Table 2). These peaks were detected with $[\mathrm{M}+\mathrm{Na}]^{+}$or $[\mathrm{M}+\mathrm{H}]^{+}$ionization. The difference in each molecular mass was $\sim 14$ Da respectively, for instance, between $\mathrm{m} / z$ 723.44, 737.45, and 751.47 as corresponding to the molecular mass of $\mathrm{CH}_{2}$ from the different ceramide composition.

\subsection{Isolation and characterization of the sphingomyelins from $\mathrm{H}$. roretzi, $\mathrm{H}$. aurantium, and $\mathrm{S}$. clava}

Sphingomyelins of 3 species of ascidians $H$. roretzi, $H$. aurantium, and $S$. clava, which belong to a different order than that of $C$. intestinalis, were isolated using the same preparation. Table 3 shows the yields at each fraction stage and the sphingomyelin content from each ascidian. The structures of the sphingomyelin were determined by the methods mentioned above. Characterization of these ceramide moieties was performed by CG and CG-MS analyses (Table 1).

\section{DISCUSSION}

Most of the phosphosphingolipid in vertebrates is in the form of sphingomyelin, but it has been thought that this is not the case in invertebrates. In fact, the phosphosphingolipid identified in invertebrates is present as ceramide phosphoethanolamine ${ }^{3-5)}$ and ceramide aminoethylphosphonate ${ }^{16-17)}$, otherwise sphingomyelin has rarely been observed. Recently, the presence of sphingomyelin has been found in vertebrates using lysenin, a sphingomyelinspecific toxin ${ }^{5,6)}$. However, there is insufficient data to decide the existence of the sphingomyelin in the invertebrates based on results from only the above analysis. It is necessary to isolate and characterize the sphingomyelin in order to understand the sphingomyelin function.

In this study, we first isolated sphingomyelin and identified its structure from the ascidian $C$. intestinalis of Enterogona. Next, we identified the sphingomyelin structure of 3 species in other ascidians Pleurogona, $H$. roretzi, $H$. aurantium, and $S$. clava, and this enabled comparative analysis of the ceramide compositions. The ceramide moiety of sphingomyelin in Enterogona and Pleurogona ascidians, comprised $\mathrm{C} 16: 0$ and $\mathrm{C} 18: 0$ fatty acids and d16:1 sphingenine and d18:2 sphingadiene; however, hydroxyl fatty 
acids were observed in Pleurogona ascidians. Thus, the major components of the ceramide moiety were similar across the species, but the minor components differed. The yields of sphingomyelin in each ascidian were also similar (Table 3).

We have identified novel acidic glycosphingolipids, sulfated glycosphingolipid (SGL-1) ${ }^{12)}$ and uronic acid-contained glycosphingolipid (UGL-1) ${ }^{13}$, and several neural glycosphingolipids (in preparations) from 2 ascidians, $C$. intestinalis (Enterogona) and $H$. roretzi (Pleurogona). The major components of the ceramide moiety of SGL-1 consisted of C16:0, 2-hydroxy-C21:0 and 2-hydroxy-C22:0 fatty acids and C16-, C17-, branched C17-, C18- and branched C18-phytosphingosines, while that of the ceramide moiety of UGL-1 consisted of C16:0 and C18:0 fatty acids and C16-, C17- and C18-phytosphingosines. The ceramide components of neutral glycosphingolipids from $C$. intestinalis and $H$. roretzi were very similar to that of acidic glycosphingolipids. Therefore, ceramide glycosyltransferase and ceramide cholinephosphotransferase could specifically recognize and/or distinguish the different ceramide components on glycosphingolipid and sphingomyelin biosynthesis.

\section{ACKNOWLEDGEMENT}

We are grateful to Prof. Takahito Nishikata of Konan University (Faculty of Science and Engineering) for the supply of $C$. intestinalis. This research was supported in part by a High-Tech Research Center Project for Private Universities: matching fund subsidy from the Ministry of Education, Culture, Sports, Science and Technology of Japan.

\section{References}

1. Jarvis, W.D.; Kolesnick, R.N.; Fornari, F.A., Traylor, R.S.; Gewirtz, D.A.; Grant, S. Induction of apoptotic DNA damage and cell death by activation of the sphingomyelin pathway. Proc. Natl. Acad. Sci. U.S.A. 270, 73-77 (1994).

2. Jayadev, S.; Liu, B.; Bielawska, A.E.; Lee, J.Y;; Nazaire, F.; Pushkareva, M.Y.; Obeid, L.M.; Hannum, Y.A. Role for ceramide in cell cycle arrest. J. Biol. Chem. 270, 2047-2052 (1995).

3. Hori, T.; Itasaka, O.; Inoue, H.; Akai, M. Occurrence of a new phosphosphingoside in the phound-snail. Heterogen longispira. J. Exp. Med. 35, 81-84 (1965).

4. Hori, T.; Itasaka, O.; Inoue, H.; Gamo, M., Arakawa, I. Biochemistry of shellfish lipids. IV. Purification and characterization of a new phosphosphingolipid in the pond-snail. Heterogen longispira. Jpn. J. Exp. Med.
36, 85-89 (1966).

5. Hori, T.; Itasaka, O.; Sugita, M.; Arakawa, I. Isolation of sphingoethanolamine from pupae of the green-bottle fly, Lucilia caesar. J. Biochem. 64, 123-124 (1968).

6. Sugita, M.; Kurimoto, A.; Dulaney, J.T.; Suzuki, M.; Suzuki, A.; Ohta, S. Sphingomyelin in the Parasitic Nematode, Ascaris sum. J. Jpn. Oil Chem. Soc. (J. Oleo Sci.) 45, 1261-1266 (1996).

7. Nakai, Y.; Sakurai, Y.; Yamaji, A.; Umeda, M.; Asou, H.; Uyemura, K.; Itoh, K. Lysenin-Sphingomyelin binding at the cell surface of oligodendrocyte linage cells increases during differentiation in vitro. J. Neurosci. Res. 62, 521-529 (2000).

8. Kobayashi, H.; Ohta, N.; Umeda, M. Biology of lysenin, a protein in the coelomic fluid of the earthworm Eisenia fetida. Int. Rev. Cytol. 236, 45-103 (2004).

9. Dehal, P.; Satou, Y.; Campbell, RK, Chapman, J.; Degnan, B.; De Tomaso, A.; Davidson, B.; Di Gregorio, A.; Gelpke, M.; Goodstein, D.M.; Harafuji, N.; Hastings, K.E.; Ho, I.; Hotta, K.; Huang, W.; Kawashima, T.; Lemaire, P.; Martinez, D.; Meinertzhagen, I.A.; Necula, S.; Nonaka, M.; Putnam, N.; Rash, S.; Saiga, H.; Satake, M.; Terry, A.; Yamada, L.; Wang, H.G.; Awazu, S.; Azumi, K.; Boore, J.; Branno, M.; Chin-Bow, S.; DeSantis, R.; Doyle, S.; Francino, P.; Keys, D.N.; Haga, S.; Hayashi, H.; Hino, K.; Imai, K.S.; Inaba, K.; Kano, S.; Kobayashi, K.; Kobayashi, M.; Lee, B.I.; Makabe, K.W.; Manohar, C.; Matassi, G.; Medina, M.; Mochizuki, Y.; Mount, S.; Morishita, T.; Miura, S.; Nakayama, A.; Nishizaka, S.; Nomoto, H.; Ohta, F.; Oishi, K.; Rigoutsos, I.; Sano, M.; Sasaki, A.; Sasakura, Y.; Shoguchi, E.; Shin-i, T.; Spagnuolo, A.; Stainier, D.; Suzuki, M.M.; Tassy, O.; Takatori, N.; Tokuoka, M.; Yagi, K.; Yoshizaki, F.; Wada, S.; Zhang, C.; Hyatt, P.D.; Larimer, F.; Detter, C.; Doggett, N.; Glavina, T.; Hawkins, T.; Richardson, P.; Lucas, S.; Kohara, Y.; Levine, M.; Satoh, N.; Rokhsar, D.S. The draft genome of Ciona intestinalis: insights into chordate and vertebrate origins. Science 298, 2157-2167 (2002).

10. Satou, Y.; Kawashima, T.; Kohara, Y.; Satoh, N. Large scale EST analysis in Ciona intestinalis: Its application as Northern blot analyses. Dev. Genes Evol. 213, 314-318 (2003).

11. Yamada, L.; Kobayashi, K.; Satou, Y.; Satoh, N. Microarray analysis of localization of maternal transcripts in eggs and early embryos of the ascidian, Ciona intestinalis. Dev. Biol. 284, 536-550 (2005).

12. Yamada, S.; Matsumuro, Y.; Inoue, T.; Kitamura, T.; Itonori, S.; Sugita, M.; Ito, M. A novel sulfatide, GlcCer-I6 sulfate, from the ascidian Ciona intestinalis. J. Oleo. Sci. 56, 129-136 (2007).

13. Ito, M.; Matsumuro, Y.; Yamada, S.; Kitamura, T.; Itonori, S.; Sugita, M. Isolation and characterization of a novel uronic acid-containing acidic glycosphingolipid 
from the ascidian Halocynthia roretzi. J. Lipid Res. 48, 96-103 (2007).

14. John, C.D.; Robert, L.L. A simple, specific spray for the detection of phospholipids on thin-layer chromatograms. J. Lipid Res. 5, 126-127 (1964).

15. Hanes, C.S.; Isherwood, F.A. Separation of the phosphoric esters on the filter paper chromatogram. Nature 164, 1107-1112 (1949).
16. Hori, T.; Arakawa, I.; Sugita, M. Distribution of ceramide 2-aminoethylphosphonate and ceramide aminoethylphosphate (sphingoehanolamine) in some aquatic animails. J Biochem. 62, 67-70 (1967).

17. Hori, T.; Horiguchi, M.; Hayashi, A. ed. Biochemistry of natural C-P compounds. Maruzen Ltd., Kyoto (1968). 